\title{
Coronary Artery Aneurysm Revealed by an Acute Coronary Syndrome in a Patient with Systemic Lupus Erythematosus: A Case Report
}

\author{
Hind Adnane*, Saousan Serbout, El Ghali Med benouna, Drighil Abdenasser, Azzouzi Leila and Habbal \\ Rachida
}

Department of Cardiology, Ibn Rochd University Hospital, Casablanca, Morocco

Correspondence should be addressed to Hind Adnane, hndadnane@ gmail.com

Received Date: June 25, 2020; Accepted Date: July 06, 2020; Published Date: July 13, 2020

\begin{abstract}
Systemic lupus erythematosus is an autoimmune inflammatory disease of unknown etiology, the coronary lesions are extremely rare, and in particular the coronary aneurysms.
\end{abstract}

We report a case of a coronary aneurysm of a patient diagnosed with Systemic lupus erythematosus who showed up at the emergency department suffering from a retro-sternal pain radiating to the jaws which he reported for 4 hours.

An electrocardiogram was performed urgently and showed an offset of $2 \mathrm{~mm}$ in the inferior, right, basal and low lateral territories with a mirror V1, V2.

The patient underwent coronary angiography which revealed an aneurysmal dilatation of the right coronary artery with multiple atheromatous aneurysms of different sizes without thrombi visualized in the lumen of the arteries.

Coronary aneurysms are a fatal and has potentially fatal complications that should be considered in the diagnostic of systemic lupus erythematosus patients with cardiac symptoms as early detection may allow complete healing.

\section{KEYWORDS}

Systemic lupus erythematosus; Coronary aneurysm; Acute coronary syndrome

\section{INTRODUCTION}

Systemic lupus erythematosus is an autoimmune inflammatory disease of unknown etiology, characterized by the presence of anti-native anti-deoxyribonucleic acid antibodies as well as multi-organ disorders including cutaneous, renal and cardiac.

The most commonly reported cardiac events are Libman-

Sacks endocarditis, myocarditis or pericarditis. The Citation: Hind Adnane, Coronary Artery Aneurysm Revealed by an Acute Coronary Syndrome in a Patient with Systemic Lupus Erythematosus: A Case Report. J Heart 1(1): 20-23.

coronary lesions are extremely rare, and in particular the coronary aneurysms, described in only $0.15 \%$ to $4.9 \%$ of reported cases.

\section{CASE REPORT}

We report through this observation a case of a coronary aneurysm of a patient diagnosed with Systemic lupus erythematosus.

Our patient is a 54-year-old; he has been chronically C2020 Tridha Scholars 
smoking for 25 years and has been treated for a lupus erythematosus in its strict cutaneous form. The patient has been monitored for 1 year and placed on synthetic antimalarial drug (Nivaquine) with poor follow up. The treatment has been stopped by the patient 2 months before his hospitalization.

This latter showed up to the emergency department suffering from an epigastralgia and retro-sternal pain radiating to the jaws which he reported for 4 hours.

He was hemodynamically stable with arterial pressure at $110 \mathrm{mmHg} / 70 \mathrm{mmHg}$, and cardiac frequency at $74 \mathrm{bpm}$, cardiopulmonary auscultation was otherwise normal.

A 12 lead derivations electrocardiogram was performed urgently and showed a regular and sinus rhythm at 70 beat per minute (bpm), a first degree atrioventricular block, with an offset of $2 \mathrm{~mm}$ in the inferior, right, basal and low lateral territories with a mirror V1, V2 (Figure 1).

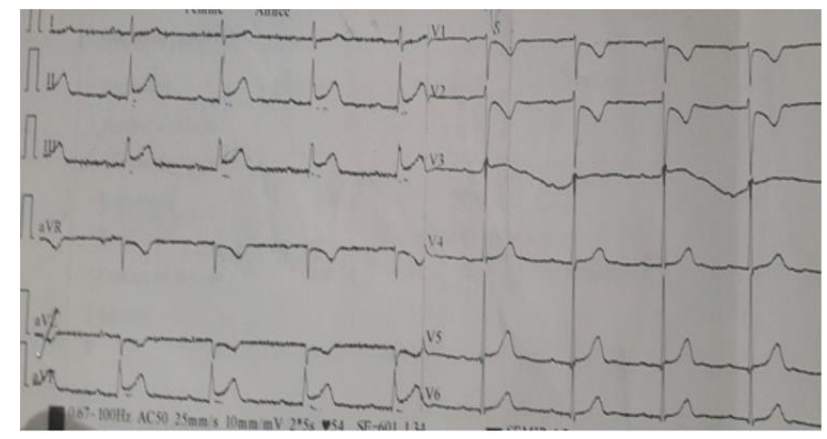

Figure 1: An offset of $2 \mathrm{~mm}$ in the inferior, right, basal and low lateral, a first degree atrioventricular block territories with a mirror V1, V2.

The patient received a loading dose of aspirin and clopidogrel, as well as low molecular weight heparin. A transthoracic echocardiography probe shot showed a mild basal and inferoseptal basal hypokinesia with preserved left ventricular ejection fraction at $60 \%$, a good longitudinal systolic function of the right ventricular and an ascending tubular aorta dilated at $41 \mathrm{~mm},\left(27 \mathrm{~mm} / \mathrm{m}^{2}\right.$ for a body surface at 1.5 ).
An initial biological assessment found Ic troponins positive at $0.17 \mathrm{ng} / \mathrm{ml}$, negative C-reactive protein, normochromic normocytic anemia at $10.9 \mathrm{~g} / \mathrm{dl}$ of hemoglobin with lymphopenia at 210 elements $/ \mathrm{mm}^{3}$.

The patient underwent coronary angiography which revealed an aneurysmal dilatation of the left communal trunk (Figure 2), and the right coronary artery with multiple atheromatous aneurysms of different sizes (Figure 3) without thrombi visualized in the lumen of the arteries.

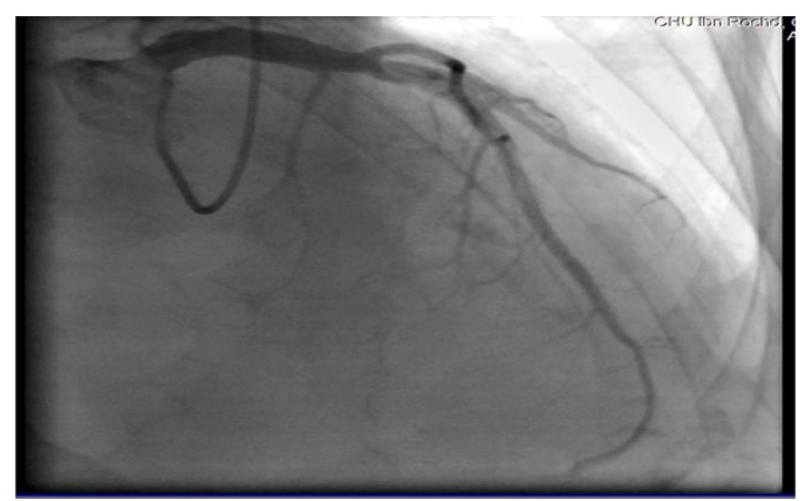

Figure 2: An aneurysmal dilatation of the left communal trunk.

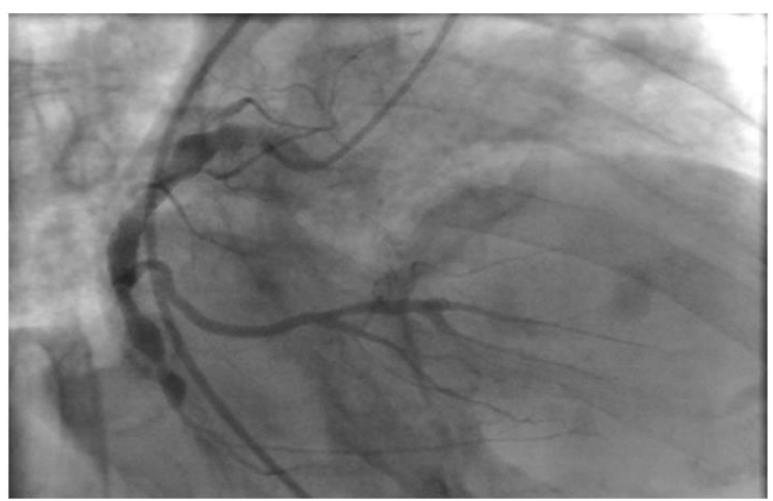

Figure 3: An aneurysmal dilatation of the right coronary artery with multiple atheromatous aneurysms of different sizes.

A requested immunoassay displayed positive anti-nuclear antibody and native anti-deoxyribonucleic acid antibodies without circulating antibodies or antiphospholipids.

We therefore retained the diagnostic of an ST elevation myocardial infarction secondary to a coronary aneurysm as part of a systemic lupus erythematosus. 
The patient was put on immuno-suppressants by the internists, a treatment based on a double anti platelet aggregation was maintained as well as a beta blocker and a statin. The patient remained asymptomatic after one year of the event.

\section{DISCUSSION}

Aneurysmal dilation of the coronary arteries has been commonly described in patients treated for polyarteritis nodosa, Takayasu's disease, and Kawasaki disease and in combination with atherosclerosis.

The prevalence of coronary aneurysms in systemic lupus erythematosus patients is unknown [1]. The process leading to the development of coronary aneurysms in these patients is not fully understood; it has been reported to be an inflammation and a sequela of necrosis of the medial tunica. Coronary aneurysm may be associated with thrombosis.

To the extent that traditional cardiovascular risk factors may play a role in the progression of coronary artery disease to coronary aneurysms, these factors should be clarified. All major epicardial coronary arteries may be involved; however, no involvement of the left main coronary artery has been reported [1]. Multiple and giant coronary aneurisms have been reported $[2,3]$.

There are no guidelines for the best management strategy for systemic lupus erythematosus patients with coronary aneurysms. The control of cardiovascular risk factors is the pillar of treatment [1].

We need to increase knowledge of lupus coronary heart disease and its complications. Coronary aneurysms are a fatal and has potentially fatal complication that should be considered in the differential of systemic lupus erythematosus patients with cardiac symptoms as early detection may allow complete healing [4-6].

This case also emphasizes the use of angiography which allowed rapid diagnosis and treatment of its condition.

\section{CONCLUSION}

Cardiac manifestations of systemic lupus erythematosus are quite common and can remain asymptomatic for a long time. Coronary aneurysm remains a very rare complication of the disease that can mimic acute coronary syndrome. The prognosis remains severe by the occurrence of a coronary insufficiency.

\section{CONFLICT OF INTEREST}

The authors declare that they have no competing interests.

\section{REFERENCES}

1. Famularo G, Vallone A, Compagnucci M, et al. (2014) Tiny bubbles: Coronary aneurysms and lupus. The American Journal of Medicine 127(10): 933-935.

2. Nobrega TP, Klodas E, Breen JF, et al. (1996) Giant coronary artery aneurysms and myocardial infarction in a patient with systemic lupus erythematosus. Catheterization and Cardiovascular Diagnosis 39(1): 75-79.

3. Matayoshi AH, Dhond MR, Laslett LJ (1999) Multiple coronary aneurysms in a case of systemic lupus erythematosus. Chest 116(4): 1116-1118.

4. Famularo G, Vallone A, Pizzicannella M, et al. (2015) Coronary aneurysms in patients with systemic lupus erythematosus. American Journal of Emergency Medicine 1(33): 120-121.

5. Harouna H, Aboudib F, Bouissar W, et al. (2016) Cardiac manifestations during systemic lupus erythematosus. The Review of Internal Medicine 37(Suppl. 2): A240.

6. Pugnet G, Baixas C, Guinault D, et al. (2012) A rare cause of coronary aneurysm: Lupus. The Journal of Internal Medicine 33(Suppl. 1): S153. 